\title{
Uma aplicação da Realidade Aumentada em Laboratórios Mistos para Ensino de Química
}

\author{
Alexandre Scotta ${ }^{1,2}$, Valquíria Hüttner ${ }^{1,2}$, Karina S. Machado ${ }^{1,2}$, Ivete Pinto ${ }^{1,2}$, Zélia \\ Couto $^{1}$, Danúbia B. Espíndola ${ }^{1,2}$
}

${ }^{1}$ Seretaria de Educação a Distância - Universidade Federal de Rio Grande (FURG) Av. Itália Km. 8 - 96.203-900 - Rio Grande - R- Brasil

${ }^{2}$ Centro de Ciência Computacionais - Universidade Federal do Grande (FURG) Av. Itália Km. 8 - 96.203-900 - Rio Grande - RS - Brasil

\begin{abstract}
This work paper aims to present a use case of Augmented Reality in virtual laboratories for chemistry teaching. In order to validate the application were proposed the creation of a augmented guide for chemical experiments. This way the teacher includes markers in chemical components in the bench and the student, with a mobile device, can view detailed information for each substance as a instruction sequence to perform the experiment step by step. Since this is a preliminary study, it is expected that future work proposing a generic architecture for creating different mixed experiments for education areas.
\end{abstract}

Resumo. Este trabalho tem por objetivo apresentar um caso de uso da Realidade Aumentada em laboratórios virtuais para ensino de química. Para validação propõem-se uma aplicação no laboratório de química, a partir da criação de um guia de instruções aumentado e autônomo para experimentos químicos. Com este recurso, o professor inclui marcadores nos componentes da bancada química e o aluno, com um dispositivo móvel, consegue visualizar informações detalhadas de cada substância assim como uma sequência de instruções de como realizar o experimento passo a passo. Sendo este um trabalho preliminar, espera-se que trabalhos futuros proponham uma arquitetura genérica para criação de diferentes experimentos para laboratórios mistos na área da educação.

\section{Introdução}

Laboratórios virtuais para auxílio de experimentos práticos são de suma importância para aplicações na Educação. Experimentos mistos com a mistura de componentes reais e virtuais para bancadas de química, física, biologia e matemática são algumas das possibilidades do uso de Realidade Aumentada (RA) [Tori et al, 2006]. Com a facilidade do uso de dispositivos móveis, a aplicação de RA em ambientes de ensino tem crescido e se tornado atrativo [Luz, 2008].

Além disso, com o crescimento dos cursos on-line a distância é cada vez mais necessário prover aos estudantes meios de realizarem atividades práticas que certifiquem e validem o aprendizado teórico do conteúdo. Os laboratórios virtuais com 
experimentos mistos permitem a experimentação científica dos alunos localizados em qualquer lugar podendo ser remotamente guiados pelos professores.

O uso da Realidade Aumentada em laboratórios virtuais, permite a sobreposição de elementos virtuais sobre o experimento real. O principal propósito do uso de Realidade Aumentada em experimentos educacionais, é manter a visualização do ambiente real e guiar remotamente o estudante na realização do experimento. Apesar de os laboratórios virtuais apresentarem diversos fatores positivos tais como: barateamento dos processos, maior segurança em experimentos com manipulação de objetos de risco, escalabilidade para acesso concorrente de experimento a partir de qualquer local, aspectos negativos também podem ser identificados tais como: tempo para desenvolvimento da aplicação, apropriação da tecnologia por parte de alunos e professores.

Dessa forma, este trabalho propõem o desenvolvimento de uma aplicação de Realidade Aumentada com marcadores através de dispositivos móveis (telefones e tablets), tendo como caso de uso o desenvolvimento de uma ferramenta para auxílio a experimentos químicos. $\mathrm{O}$ artigo organiza-se da seguinte forma: a seção 2 descreve o resumidamente o referencial teórico e trabalhos relacionados. A seção 3 descreve a metodologia proposta e a seção 4 o caso de uso em desenvolvimento. A seção 5 apresenta as considerações finais.

\section{Referencial teórico e trabalhos relacionados}

Na Realidade Aumentada o ambiente é predominante real e objetos virtuais são trazidos à cena para auxiliar na visualização [Milgram, et. al.,1994]. A Realidade Aumentada "consiste em inserir elementos virtuais em cenas reais, de modo que eles possam coexistir. Além disso, o usuário poderá interagir em tempo real com os objetos reais e virtuais." [Silva, 2012]. A sobreposição de elementos virtuais pode ser realizada através de diferentes técnicas, entre elas o uso de marcadores. A RA por meio de marcadores é capaz de "apresentar os objetos ou informações virtuais no espaço real através do reconhecimento de padrões na cena" [Anami, 2013].

Uma das bibliotecas de Realidade Aumentada de maior utilização no meio acadêmico é a biblioteca ARToolkit. No trabalho de Cunha, uma interface gráfica para utilização da biblioteca ARToolkit com fins educacionais é apresentada [Cunha e Guimarães, 2007]. "O funcionamento do ARToolKit baseia-se no uso de marcadores através de técnicas de visualização computacional para calcular a posição da câmera e sua orientação em relação a estes marcadores. Os marcadores geralmente são etiquetas de papel com um símbolo impresso em uma moldura quadrada” [Rodrigues, 2010].

\section{Metodologia}

Para o desenvolvimento deste trabalho estão sendo utilizadas tecnologias para a Web como HTML, CSS e Javascript. Como biblioteca de Realidade Aumentada esta sendo utilizado o JARTookit que é a implementação em Java da biblioteca ARToolkit. 
A metodologia para o desenvolvimento do trabalho propõem a seguinte sequência de etapas:

(i) Instalação de biblioteca de Realidade Aumentada para sistema Android com execução em navegador;

(ii) Cadastramento de marcadores e registro das informações relacionadas aos mesmos;

(iii) Definição das funcionalidades e criação da interface do aplicativo;

(iv) Implementação do aplicativo para uso em dispositivos móveis com suporte ao Sistema Operacional Android.

\section{Aplicação}

De forma a validar a metodologia foi desenvolvido uma aplicação utilizando marcadores sobre componentes de uma bancada química. A idéia é utilizar a Realidade Aumentada como guia de instruções no experimento laboratorial. A seqüência e descrição dos passos do experimento é dado pela colocação dos marcadores nos frascos e programação do aplicativo.

Como primeiro passo três tubos de ensaio utilizados em um experimento foram utilizados no laboratório de Química da Universidade Federal do Rio Grande (FURG) de forma a simular o ambiente e realizar o protótipo de interface. Em seguida foi realizado uma breve entrevista com professor de química a fim de registrar um experimento de mistura de três elementos químicos. A sequencia de mistura e as informações químicas sobre cada elemento foram registradas para posterior apresentação e integração no aplicativo.

Definida a aplicação e registrado os passos e descrição do experimento, foi desenvolvido um protótipo de interface sobre a imagem do ambiente real. Foram colocados marcadores em cada tubo de ensaio para poder acrescentar os elementos e informações virtuais sobre o ambiente. O protótipo de interface é apresentado na Figura 1. Ícones para inserção de funcionalidades também são apresentados.

O protótipo apresenta um botão de captura de imagem em vermelho (representado pelo ícone de uma câmera - Figura 1), que quando pressionado começa a executar o algoritmo de captura dos marcadores na cena real. Após pressionado, é ativado o botão de próximo passo, que fica logo abaixo do botão de captura. O botão de avançar vai apresentando os passos do experimento e indicando os tubos que devem ser trabalhados e as informações a respeito de componentes e quantidades químicas do elemento a serem colocados no frasco. Na parte inferior da interface uma caixa de texto apresenta a descrição de cada passo do experimento. Em cada marcador é apresentado um número virtual para a definição da sequencia de passos do experimento, ao pressionar o número, a caixa de texto descreve as informações a respeito dos elementos químicos que o frasco contém. 


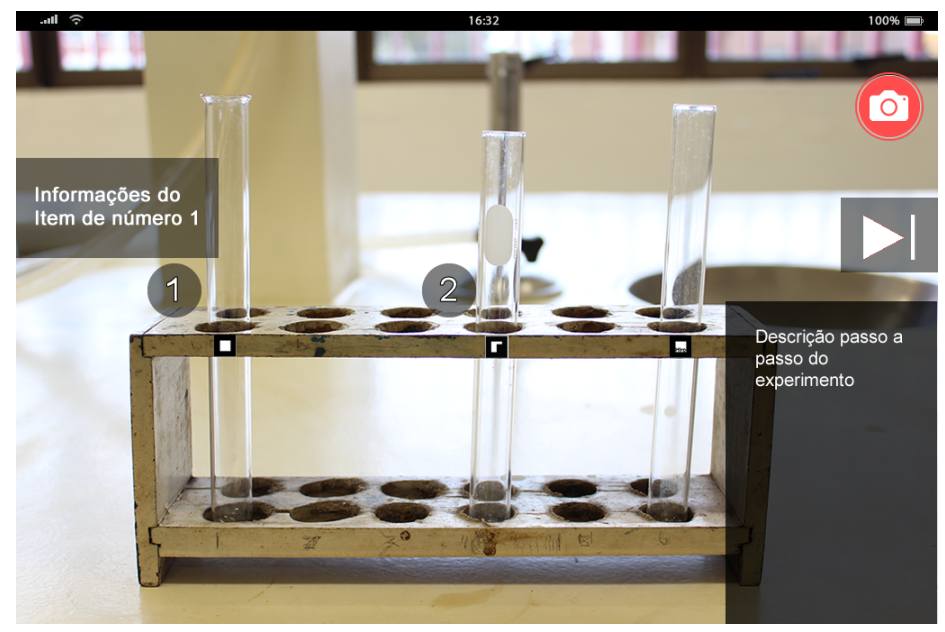

Figura 1. Protótipo do guia de instruções para experimentos de química.

\section{Considerações finais}

Apesar do estudo tratar o uso de Realidade Aumentada para experimentos químicos, estas implementações servirão de base para a proposta de uma arquitetura genérica para o uso de RA como guia remoto de experimentos mistos para suporte aos laboratórios virtuais. Através da combinação de elementos virtuais com os componentes reais em bancadas físicas é possível seqüenciar experimentos e guiar os estudantes durante a realização do mesmo.

\section{Referências}

Anami, B. M. (2013) "Boas Práticas de Realidade Aumentada Aplicada à Educação”. Londrina - PR. Pages $1-51$.

Cunha, K. K. C.; Guimarães, M. P. (2007) "Desenvolvimento de uma Interface Gráfica para o Artoolkit com Aplicação na Área Educacional”. WRVA'07 Workshop de Realidade Virtual e Aumentada, Itumbiara. Pages 1-4.

Luz, M., Garcia, L. F. F., Machioro, G. F. (2008) "Realidade Aumentada em Dispositivos Móveis”. In: V Workshop de Realidade Virtual e Aumentada, Bauru - SP. Pages. 1 - 6

Milgram, P. et al. Augmented reality: A class of displays on the reality-virtuality continuum. In: Telemanipulador and Telepresence Technologies, Boston. London: Springer-Verlag, v.2351, n.1, 1994a. p. 282-292.

Rodrigues, C. S. C., Pinto, R. A. M., Rodrigues, P. F. N. (2010) "Uma aplicação de realidade aumentada no ensino de modelagem dos sistemas estruturais". Revista Brasileira de Computação Aplicada (ISSN 2176-6649), Passo Fundo, Pages 81-95.

Silva, M. M. O., Roberto, R. A., Teichrieb, V. (2012) "Um Estudo de Aplicações de Realidade Aumentada para Educação". In: IX Workshop de Realidade Virtual e Aumentada, Paranavaí - PR. pages. $1-6$

Tori, R., Kirner, C., Siscoutto, R. (2006). "Livro do pré-simpósio, VIII Symposium on Virtual Reality”. Editora da Sociedade Brasileira de Computação (SBC). Belém-PA. 